\title{
The Preventive Effects of Nanopowdered Peanut Sprout-added Caciocavallo Cheese on Collagen-induced Arthritic Mice
}

\author{
Dong-Hwi Kim, Yoon Hyuk Chang ${ }^{1}$, and Hae-Soo Kwak* \\ Department of Food Science and Technology, Sejong University, Seoul, 143-747, Korea \\ ${ }^{1}$ Department of Food and Nutrition, Kyung Hee University, Seoul 130-701, Korea
}

\begin{abstract}
The present study was carried out to investigate the effects of nanopowdered peanut sprout-added Caciocavallo cheese (NPCC) on the prevention and treatment of rheumatoid arthritis in DBA/IJ mice immunized with type II collagen. After the induction of arthritis, the mice were being divided into five groups: (1) normal, no immunization; (2) CIA, collagen-induced arthritis; (3) MTX, collagen-induced arthritis treated with methotrexate ( $0.3 \mathrm{mg} / \mathrm{kg}$ body weight); (4) CC, collagen-induced arthritis treated with Caciocavallo cheese $(0.6 \mathrm{~g} / \mathrm{d})$; (5) NPCC, collagen-induced arthritis treated with nanopowdered peanut sprout-added Caciocavallo cheese $(0.6 \mathrm{~g} / \mathrm{d})$. Nanopowdered peanut sprout was ranged from 300 to $350 \mathrm{~nm}$, while regular powdered peanut sprouts were ranged from 50 to $150 \mu \mathrm{m}$. The NPCC group had considerable reductions of clinical scores and paw thicknesses at the end of experiment as compared to the CIA group. In the serum analysis, the TNF- $\alpha$, IL- $1 \beta$, IL6 and $\mathrm{IgG}_{1}$ levels in the NPCC group have decreased by $69.4,75.9,66.6$, and $61.9 \%$, respectively, when compared to the CIA group. The histological score and spleen index of the NPCC group were significantly lower than the CIA group. In conclusion, the feeding NPCC method could delay and/or prevent the rheumatoid arthritis in the collagen-induced arthritis mouse model. Based on this study, nanopowdered peanut sprouts could be applied to various functional cheeses.
\end{abstract}

Key words: rheumatoid arthritis, nanopowdered peanut sprout, Caciocavallo cheese

\section{Introduction}

Rheumatoid arthritis is a systemic autoimmune illness characterized by chronic inflammation of synovial joints leading to destruction of cartilage and bone, and affects about $1 \%$ of world's total population (Borchers et al., 2004; Chunxia et al., 2011; Maini and Taylor, 2000; Sanchez-Fidalgo et al., 2010; Stamp et al., 2005). A great number of patients suffering from rheumatoid arthritis have been looking for alternative or complementary therapies of which diet is one of the most important factors.

Nowadays, peanut sprouts are obtained from the germination of peanut kernels, and have usually been utilized in the diet as a health food in Asia (Lee et al., 2013; Xiong et al., 2014). According to Burns et al. (2002) and Xiong et al. (2014), peanut sprouts are rich in flavonoid and resveratrol which can contribute to disease preventive and health promoting properties, such as various cancers,

\footnotetext{
*Corresponding author: Hae-Soo Kwak, Department of Food Science and Technology, Sejong University, Seoul 143-747, Korea. Tel: 82-2-3408-3226, Fax: 82-2-3408-4319, E-mail: kwakhs@, sejong.ac.kr
}

diabetes mellitus, aging, cardiovascular disease, and so on. Wang et al. (2005) reported that the resveratrol contents of the sprouts germinated from a variety of peanuts ranged from 11.7 to $25.7 \mu \mathrm{g} / \mathrm{g}$. In particular, Elmani et al. (2006) investigated the effects of resveratrol on arthritis in the rabbit model. According to them, decreased cartilage destruction was determined after injecting $10 \mu \mathrm{mol} /$ $\mathrm{kg}$ resveratrol in DMSO into the right knees of the rabbit. Recently, Xuzhu et al. (2013) found that after 23 d treatment of resveratrol $(20 \mathrm{mg} / \mathrm{kg})$, the collagen-induced arthritis in the mice was significantly reduced when they evaluated the disease incidence, number of involved paws, footpad thickness, and clinical index.

In recent years, nanotechnology has captured the attention of researchers, manufacturers, and the general population (Park et al., 2007; Rasenack and Muller, 2004). According to Park et al. (2007), nanocalcium supplementation in milk may be an effective way to improve bone calcium metabolism for ovariectomized rats. Due to the advantage of size effect and high surface reactivity of nanoparticles, nanotechnology is already used in pharmaceutical applications to increase the bioavailability of drugs. However, there are a few reports on applications of 
nanotechnology in food industry, especially in dairy products. For example, in our previous study (Seo et al., 2009), we investigated the physicochemical, microbial, and sensory properties of yogurt supplemented with nanopowdered chitosan during storage.

Caciocavallo cheese is a semi-hard cheese and is originally manufactured in the Palermo province (Sicily, Italy). So-called healthy foods, particularly those with nutraceutical properties, are in great demand in our health conscious society. Nutraceutical Caciocavallo cheese could be a good vehicle in this respect if nutraceutical ingredients, such as nanopowdered peanut sprouts, were to be added into the cheese. However, there is no report in the literature on the production of nanopowdered peanut sprouts-added Caciocavallo cheese. Furthermore, the effects of Caciocavallo cheese or nanopowdered peanut sproutsadded Caciocavallo cheese on prevention and treatment of rheumatoid arthritis have not been examined yet. Therefore, the objective of the present study was to elucidate the effects of nanopowdered peanut sprouts-added Caciocavallo cheese on the reduction of rheumatoid arthritis in DBA/IJ mice immunized with type II collagen.

\section{Materials and Methods}

\section{Materials}

Powdered peanut sprouts were offered by Jangsuche. Co., Ltd. (Korea). Nanopowdered peanut sprouts by the dry milling was supplied from Apexel Co. (Korea).

\section{Particle size analysis}

The particle size analysis was measured according to the procedure of Seo et al. (2009). Powdered peanut sprouts or nanopowdered peanut sprouts was mounted on a brass stub (10 $\mathrm{mm}$ in diameter) using two-sided adhesive tape. The stub surface was gently blown to remove unattached peanut sprout powders using a hand-held blower. The specimens were then made electrically conductive by coating under an argon atmosphere with a thin layer (approximately $30 \mathrm{~nm}$ in thickness) of platinum-palatium (8:2). The specimens were examined using a scanning electron microscope (SEM, Hitachi S-4700, Japan) operated at an accelerating voltage of $15 \mathrm{kV}$. The particle size of nanopowdered peanut sprouts was determined by Delsa (TM) Nano particle size analyzer (Beckman Coulter, USA).

\section{Production of nanopowdered peanut sprouts-added Caciocavallo cheese}

Caciocavallo cheese was produced using the method described by Gobbetti (2002). For the manufacture of nanopowdered peanut sprouts-added Caciocavallo cheese, $0.5 \%(\mathrm{w} / \mathrm{v})$ of nanopowdered peanut sprouts was added into the raw milk. After pasteurization (LTLT) nanopowdered peanut sprouts-added milk was cooled down to $32^{\circ} \mathrm{C}$. One percentage of a frozen concentrated starter culture (ABT-5, Chr. Hansen's Laboratory) was added to the milk. After adding $0.02 \% \mathrm{CaCl}_{2}$, the milk was set for $1 \mathrm{~h}$ and $0.025 \%$ rennet (double strength 290, Chr. Hansen's Laboratory) was added to the milk sample. Milk was then stirred quickly for $3 \mathrm{~min}$ and set to coagulate at 32 to $33^{\circ} \mathrm{C}$ for $45 \mathrm{~min}$, and the curd was cut to size of $0.8 \times 0.8 \times 0.8$ $\mathrm{cm}$ and the curd-whey mixture was gradually stirred during increasing to $48^{\circ} \mathrm{C}$ to cook for $30 \mathrm{~min}$ and $\mathrm{pH}$ reached to 5.8. After stirring, whey was drained as much as possible and the curd was relocated to one side of the vat. Matting the curds was done till reaching $\mathrm{pH} 5.2$ and cut to 0.3 $\mathrm{mm}$ curd was stretched at $80^{\circ} \mathrm{C}$. The stretched curds were shaped to a gourd dipper, $20 \%$ brine solution at $12^{\circ} \mathrm{C}$ for $1-2 \mathrm{~h}$ and ripened at $14^{\circ} \mathrm{C}$ for $6 \mathrm{wks}$ at a relative humidity of $98 \%$. Control and sample cheeses were made in duplicate in 2 batches.

\section{Animals}

Male DBA/1J mice ( $5 \mathrm{wks}$ old; $n=30$ ) were purchased from Central Lab. Animal Inc. (Korea). The mice were housed in plastic cages under the constant temperature of $25 \pm 3^{\circ} \mathrm{C}$, and the constant humidity of $55 \pm 5 \%$ on $12 \mathrm{~h}$ light/dark cycle. Experimental diets were based on the AIN-76A diet (American Institute of Nutrition, 1977), as shown in Table 1. All the animal treatments and proce-

Table 1. Composition of AIN-76A Purified diet

\begin{tabular}{cc}
\hline \hline Ingredient & $\%$ \\
\hline Casein & 20.0 \\
DL-Methionine & 0.3 \\
Cornstarch & 15.0 \\
Sucrose & 50.0 \\
Fiver & 5.0 \\
Con oil & 5.0 \\
AIN Mineral mix & a \\
AIN Vitamin mix & 3.5 \\
Choline bitartrate & 1.0 \\
\hline
\end{tabular}

a AIN-76 mineral mix (ing/kg): $\mathrm{CaHPO}_{4}, 500 ; \mathrm{NaCl}, 74$ potassium citrate monohydrate, $220 ; \mathrm{K}_{2} \mathrm{SO}_{4}, 52 ; \mathrm{MgO}, 24 ; \mathrm{Mn}$ carbohydrate, 3.5; Fe citrate, 6.0; $\mathrm{Zn}$ carbonate, 1.6; $\mathrm{Cu}$ carbonate, 0.3; $\mathrm{KIO}_{3}$, $0.01 ; \mathrm{Na}_{2} \mathrm{SeO}_{4} \cdot \mathrm{H}_{2} \mathrm{O}, 0.01 ; \mathrm{CrK}\left(\mathrm{SO}_{4}\right) \cdot \mathrm{H}_{2} \mathrm{O}, 0.55 ;$ sucrose, 118 . ${ }^{b}$ AIN76 vitamin mix (in $/ \mathrm{kg}$ ): thiamine $\cdot \mathrm{HCl}, 0.6$; riboflavin, 0.6 ; pyridoxine $\mathrm{HCl}, 0.7$; nicotinic acid, 3; D-calcium pantothenate, 1.6; folic acid, 0.2; D-biotin, 0.02; cyanocobalamin, 0.001; retinylpalmitate, 0.8; DL- $\alpha$-tocopheryl acetate, 20; cholecalciferol, 0.00025; menaquinone, 0.005 . 
dures were done according to the protocol of the institutional guidelines (The Institutional Animal Care and Use Committee, IACUC) at Sejong University (Korea).

\section{Induction of collagen-induced arthritis and treat- ment}

Induction of arthritis was followed by the procedure of Brand et al. (2007). Briefly, the mice were immunized intradermally on the base of the tail with $100 \mu \mathrm{m}$ Bovine type II collagen (Sigma, USA) emulsified in Freund's complete adjuvant. On day 21, all mice were boosted with an intradermal injection [100 $\mu \mathrm{m}$ type II collagen emulsified in Freund's incomplete adjuvant (Gibco BRL, USA)]. After the induction of arthritis, the mice were divided into the following five groups depending on their diets: (1) normal, no immunization; (2) CIA, collagen-induced arthritis; (3) MTX, collagen-induced arthritis treated with methotrexate $(0.3 \mathrm{mg} / \mathrm{kg}$ body weight); (4) CC, collagen-induced arthritis treated with Caciocavallo cheese $(0.6 \mathrm{~g} / \mathrm{d})$; (5) NPCC, collagen-induced arthritis treated with nanopowdered peanut sprouts-added Caciocavallo cheese $(0.6 \mathrm{~g} /$ d). During the 18 wks experimental periods, all mice were daily fed with $0.6 \mathrm{~g}$ of NPCC mixed in feed and allowed access to diets and distilled water ad libitum.

\section{Measurement of clinical score and paw thickness}

Clinical scores were measured according to the degree of inflammation: $0=$ represented normal; grade $1=$ swelling of one finger; grade 2 = swelling of more than two finger; grade 3 = swelling of heel; grade $4=$ joint deformity with ankylosis (Brand et al., 2007). The paw thickness in the hind paw was measured using a plethysmometer 7150 (Italy) from the initial injection day. Clinical scores and hind paw thickness were monitored every other wk for a period of 18 wks.

\section{Biochemical analysis in serum}

On the final day of the experiment, all of the mice were anesthetized with ethyl ether. The blood was collected from each by abdominal aorta and was placed into the serum separation tubes. After clotting at room temperature using serum separated tube, the serum was collected by centrifuging at $4000 \times \mathrm{g}$ for $15 \mathrm{~min}$. All serums were stored at $-80^{\circ} \mathrm{C}$ until required. Cytokines [TNF- $\alpha$ (tumor necrosis factor $\alpha$ ), IL-1 $\beta$ (interleukin-1 $\beta$ ), and IL-6 (interleukin-1 $\beta)$ ] and immunoglobulin G1 were measured by Luminex fluorescent bead mouse cytokine immuno assays (MILLIPLEX MAP, Millipore Corp., USA).

\section{Histological assessment - H\&E staining}

After the mice were sacrificed, their right knee joints were collected and fixed in $10 \%$ natural buffered formalin. Tissues were decalcified in 10\% ethylene diamine tetra acetate (EDTA) for $7 \mathrm{~d}$ and then embedded in a paraffin block. Tissue sections of $4 \mu \mathrm{m}$ thickness were stained with hematoxylin and eosin (H\&E staining). Photographs of the sections were taken using an Olympus IX70 (Japan) at the magnification of $\times 100$.

According to the procedure of Mankin et al. (1971), the grade for histological changes was measured as follows: 0 , normal; 1 , surface irregularities; 2 , pannus and surface irregularities; 3 , clefts to transitional zone; 4 , clefts to radial zone; 5 , clefts to calcified zone; 6 , complete disorganization.

\section{Measurement of the spleen index}

At the end of the experiment, all mice were sacrificed by decapitation. All the spleens of mice were weighed immediately after dissection. The spleen indexes were calculated by using the following formula as described previously (Chunxia et al., 2011):

$$
\text { Spleen index }=\frac{\frac{\text { spleen weight of CIA mice }}{\text { body weight of CIA mice }}}{\frac{\text { spleen weight of normal mice }}{\text { body weight of normal mice }}}
$$

\section{Statistical analysis}

The results were expressed as mean \pm SD. Statistical significance was determined by analysis of variance and subsequent Duncan's multiple range test $(p<0.05)$. The analysis was performed using SAS statistical software.

\section{Results and Discussion}

\section{Particle size analysis}

The morphology of powdered peanut sprouts and nanopowdered peanut sprouts was observed by SEM, as shown in Fig. 1. The SEM images demonstrated that the particle size of powdered peanut sprouts apparently decreased during the manufacture of nanopowdered peanut sprouts. The average particle sizes of powdered peanut sprouts and nanopowdered peanut sprouts measured were about $50-150 \mu \mathrm{m}$ (as measured by SEM) and about 300$350 \mathrm{~nm}$ in diameter (as measured by the particle size analyzer), respectively (Fig. 1 and 2).

Measurement of clinical score and hind paw thickness The changes in the incidence and severity of arthritis in 


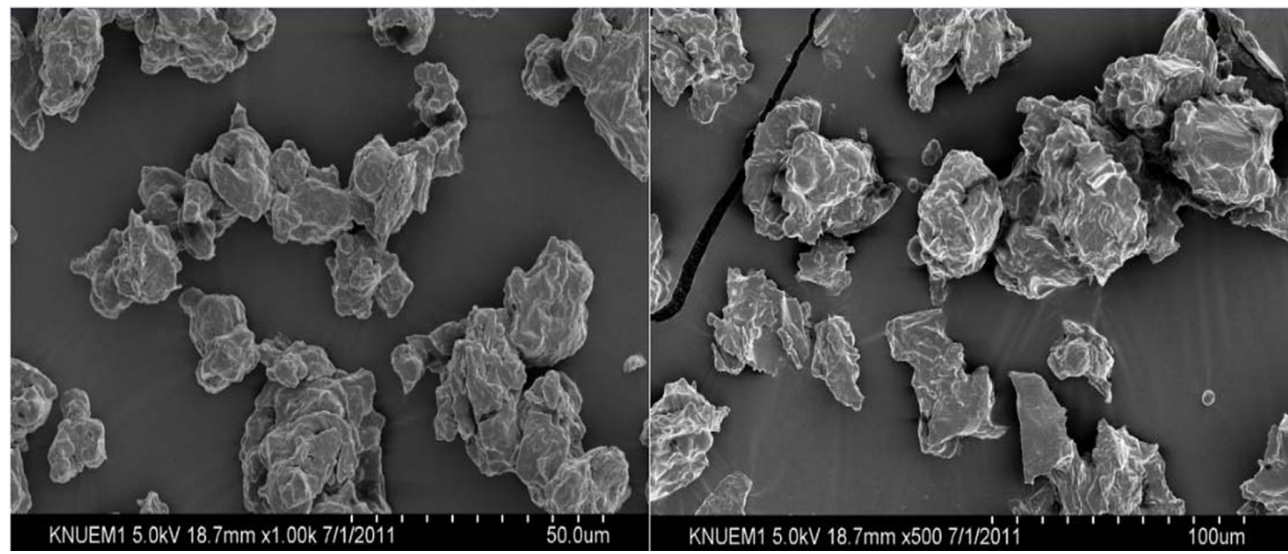

Fig. 1. Scanning electron microscope image for nanopowdered peanut sprout and powdered peanut sprouts.

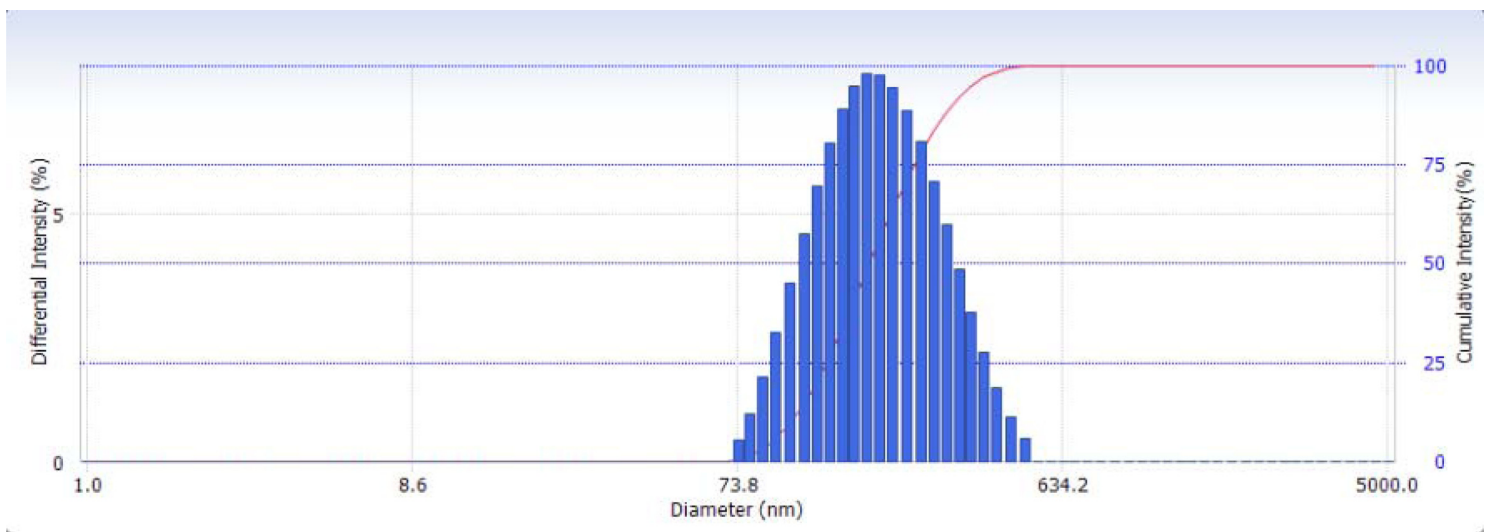

Fig. 2. Particle size analysis of nanopowdered peanut sprouts.

CIA mice treated with nanopowdered peanut sproutsadded Caciocavallo cheese during 18 wks were shown in Fig. 3. For the all the groups, except for normal, the first sign of arthritis was observed at $2^{\text {nd }}$ and $4^{\text {th }}$ wks after immunization. The clinical arthritis scores for CIA, MTX, $\mathrm{CC}$, and NPCC groups were dramatically increased until 6 wks and then the scores were not considerably changed. After $18 \mathrm{wks}$, the final clinical arthritis scores for CIA, MTX, CC, and NPCC were 3.39, 1.96, 2.79, and 2.67, respectively. This finding indicated that feeding nanopowdered peanut sprouts-added Caciocavallo cheese into the arthritic mice can reduce the incidence and severity of arthritis in mice with collagen-induced arthritis.

The values of the hind paw thickness for the MTX, CC, and NPCC groups were remarkably lower than that of the CIA group (Fig. 3). However, there was no considerable difference between CC and NPCC groups.

\section{Level on TNF- $\alpha$, IL-1 $\beta$, IL-6, and IgG1}

In general, rheumatoid arthritis is a chronic, inflammatory, autoimmune disorder of the joints, for which current treatment strategies remain suboptimal (Smolen and Steiner, 2004). Although the pathogenesis of the disease has not been fully understood, it contains cellular infiltration into synovial tissue and elevated inflammatory cytokines that lead to cartilage and bone erosion through the induction of matrix metallo proteinases (MMPs) and dysregulated chondrocyte/osteoclast function (Feldmann et al., 1996; Goldring, 2003). Among the inflammatory cytokines, TNF$\alpha$ seems to be on the top of a cytokine cascade, because it increases the levels of interleukin IL 1 $\beta$, and IL 6 (Goldring, 2003). According to Miani and Taylor (2000), TNF$\alpha$ and IL-1 play major roles in the maintenance of chronic inflammation and/or tissue damage during the progression of rheumatoid arthritis. Furthermore, IL-6 is important inflammatory cytokine in rheumatoid arthritis, and it activates auto reactive T-cell, facilitates to produce rheumatoid arthritis factors, and derives to produce acute phase reactant,consequently affecting rheumatoid arthritis.

In the present study, the serum was collected from abdominal vein of DBA/IJ mice immunized with type II collagen. The TNF- $\alpha$, IL-1, and IL-6 levels in the serum of the 

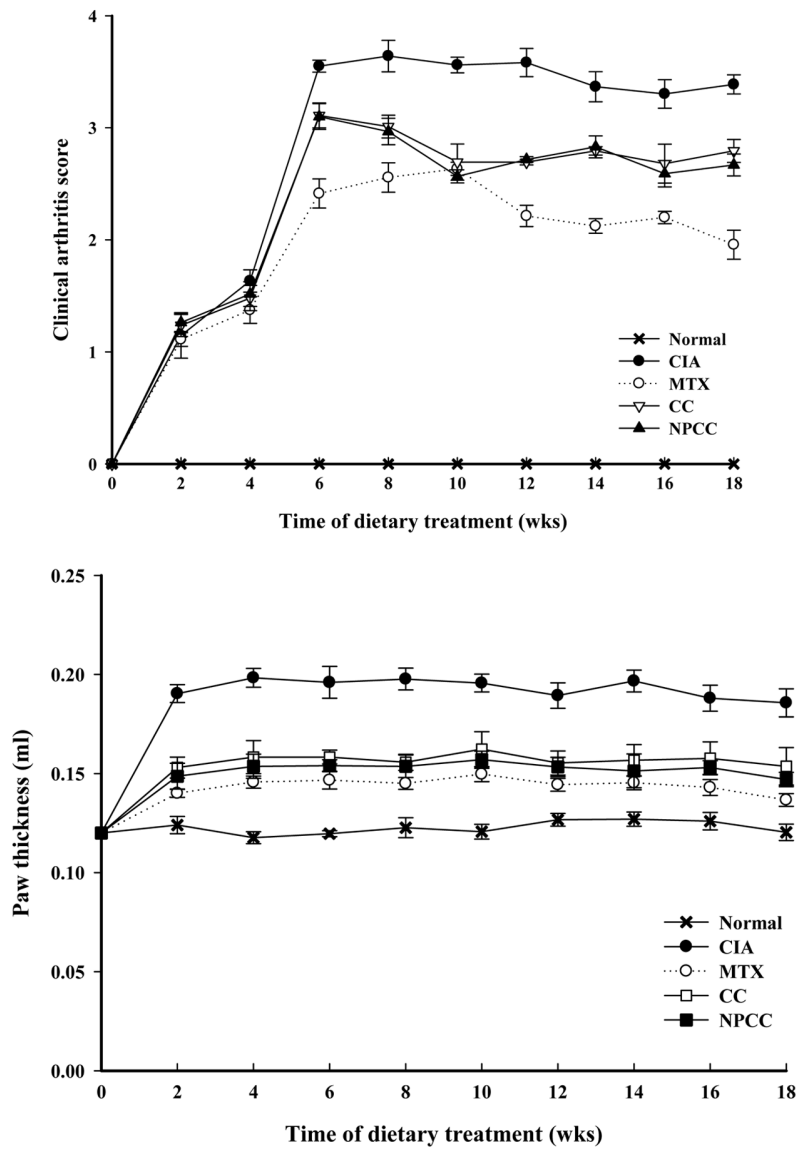

Fig. 3. Effects of Caciocavallo cheese or nanopowdered peanut sprouts-added Caciocavallo cheese on clinical scores of arthritis symptoms and hind paw thickness in arthritic mice: normal, no immunization; CIA, collagen-induced arthritis; MTX, collagen-induced arthritis treated with methotrexate; $\mathrm{CC}$, collagen-induced arthritis powdered peanut sprouts treated with Caciocavallo cheese; NPCC, collagen-induced arthritis treated with nanopowdered peanut sprouts-added Caciocavallo cheese.

arthritis mice were analyzed by Luminex ${ }^{\mathrm{TM}}$ system and are presented in Fig. 4. The methotrexate is classified as an antimetabolite drug and is used to treating rheumatoid arthritis (Borchers et al., 2004). Feeding methotrexate or nanopowdered peanut sprouts-added Caciocavallo cheese into the arthritis mice significantly decreased all of the TNF- $\alpha$, IL- $1 \beta$, and IL- 6 values as compared to the CIA group. Thus, the present study could offer the possibility of using nanopowdered peanut sprouts-added Caciocavallo cheese to reduce the symptom of rheumatoid arthritis.

The IgG1 levels in the serum of arthritis mice were presented in Fig. 5. The order for IgG1 levels in the arthritis mice groups was as follows: CIA $>$ CC $>$ NPCC $>$ MTX. The IgG1 is one of most crucial rheumatoid arthritis factors and has been used for diagnosing rheumatoid arthritis. According to Xuzhu et al. (2013), IgG1 is mainly in-

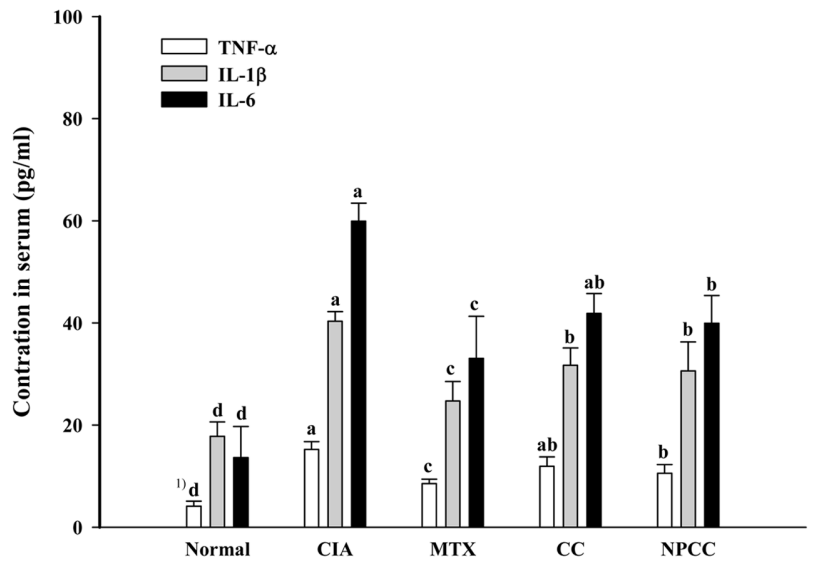

Fig. 4. Effect of Caciocavallo cheese or nanopowdered peanut sprouts-added Caciocavallo cheese on TNF- $\alpha$, IL-1 $\beta$, and IL-6 in the serum of arthritis mice: normal, no immunization; CIA, collagen-induced arthritis; MTX, collagen-induced arthritis treated with methotrexate; $\mathrm{CC}$, collagen-induced arthritis powdered peanut sprouts treated with Caciocavallo cheese; NPCC, collagen-induced arthritis treated with nanopowdered peanut sprouts-added Caciocavallo cheese. ${ }^{1)}$ Values with different superscripts are significant at $p<0.05$ by Duncan's multiple range test.

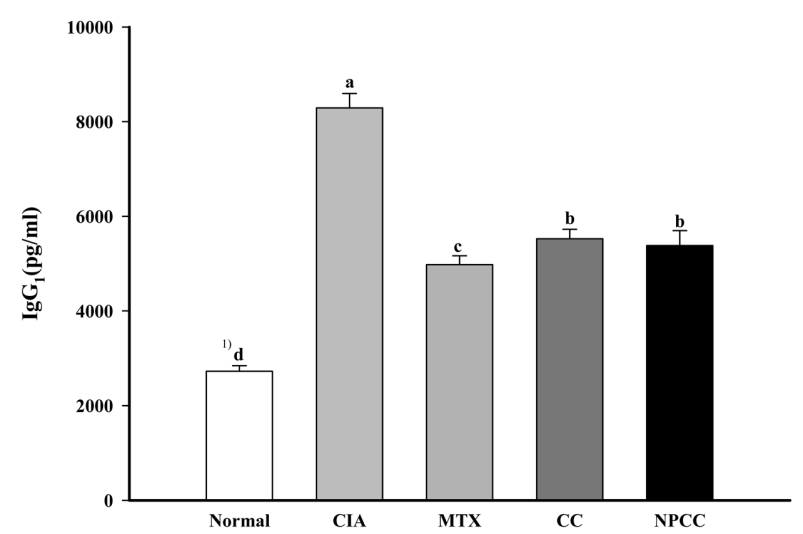

Fig. 5. Effect of Caciocavallo cheese or nanopowdered peanut sprouts-added Caciocavallo cheese on IgG1 in the serum of arthritis mice: normal, no immunization; CIA, collagen-induced arthritis; MTX, collagen-induced arthritis treated with methotrexate; CC, collagen-induced arthritis powdered peanut sprout treated with Caciocavallo cheese; NPCC, collagen-induced arthritis treated with nanopowdered peanut sprouts-added Caciocavallo cheese. ${ }^{1)}$ Values with different superscripts are significant at $p<0.05$ by Duncan's multiple range test.

duced by type II cytokines (IL-4, IL-13). The decrease in the levels of IgG1 secreted from the body is a signal of decreased disease pathology (Viji et al., 2010). Accordingly, the finding obtained from the present study on the reduction of the IgG1 level in the NPCC group may be an indication of attenuating rheumatoid arthritis in mice. 


\section{Histological analysis}

Histological analysis was carried out by $\mathrm{H} \& \mathrm{E}$ staining for the hind knee joints of DBA/1J mice immunized with type II collagen (Fig. 6(A) and 6(B)). The normal group exhibited common articular cartilage, absence of infiltrate in the synovium, and open joints pace. For the CIA group, the hind knee joints showed the typical and pathological changes with severe degrees of synovial inflammation and fibrosis, cartilage erosin, bone erosin, bone inflammation, fibrosis, and severe narrow joint space. On the other hand, the MTX and NPCC groups showed mild degrees of erosin, inflammation, and fibrosis in the hind knee joints of the mice, indicating the reduction of arthritis symptom in the mice.
Histological scores of the MTX and NPCC groups were significantly lower than that of the CIA group. Elmali et al. (2007) also reported that the dietary supplementation of resveratrol can suppress inflammatory arthritis in a rabbit model. This finding from Elmali et al. (2007) suggested that the significant decrease in the histological score for NPCC group in the present study could be related to the presence of resveratrol in nanopowdered peanut sprouts-added Caciocavallo cheese.

\section{Spleen index}

For measuring the spleen index of all the groups, the weights of spleen and body were recorded at the final day of experiment (Fig. 7). The spleen has central roles in the

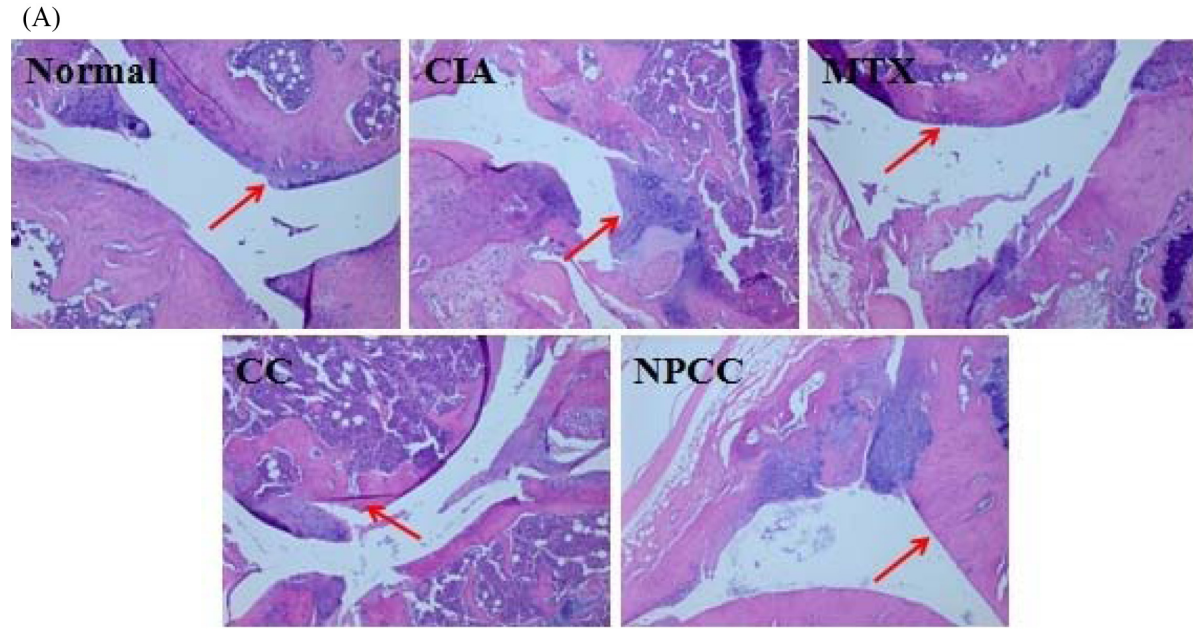

(B)

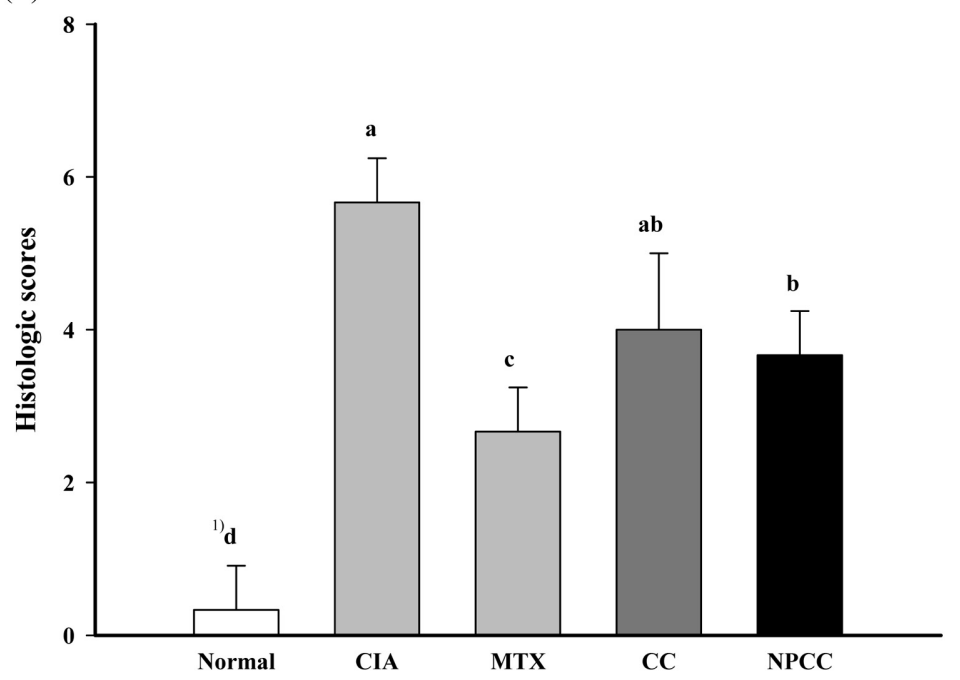

Fig. 6. Effect of Caciocavallo cheese or nanopowdered peanut sprouts-added Caciocavallo cheese on histological changes (A) and histological scores (B) in right knee joint of arthritic mice: normal, no immunization; CIA, collagen-induced arthritis; MTX, collagen-induced arthritis treated with methotrexate; $\mathrm{CC}$, collagen-induced arthritis powdered peanut sprout treated with Caciocavallo cheese; NPCC, collagen-induced arthritis treated with nanopowdered peanut sprouts-added Caciocavallo cheese. The arrows in A showed the change of bone structure. ${ }^{1)}$ Values with different superscripts are significant at $p<0.05$ by Duncan's multiple range test. 


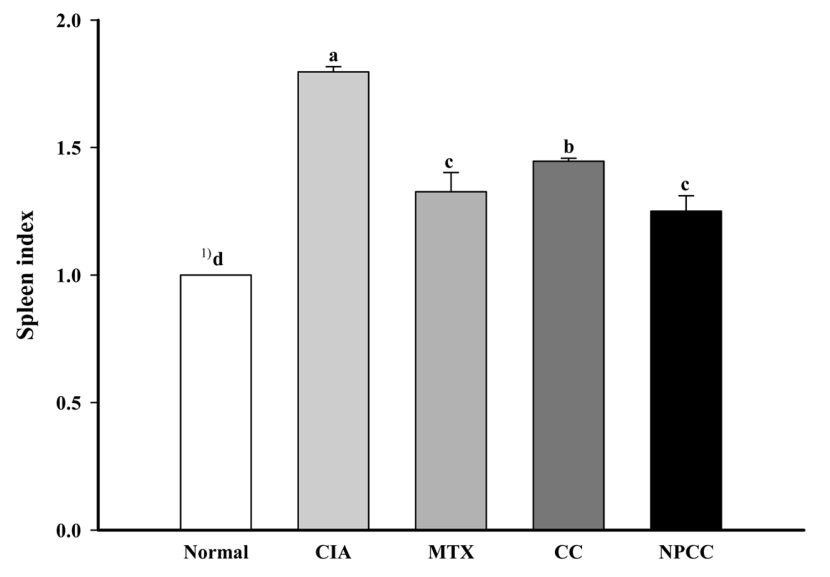

Fig. 7. Effect of Caciocavallo cheese or nanopowdered peanut sprouts-added Caciocavallo cheese on spleen indexesin arthritic mice: normal, no immunization; CIA, collageninduced arthritis; MTX, collagen-induced arthritis treated with methotrexate; $\mathrm{CC}$, collagen-induced arthritis powdered peanut sprouts treated with Caciocavallo cheese; NPCC, collagen-induced arthritis treated with nanopowdered peanut sprouts-added Caciocavallo cheese. ${ }^{1)}$ Values with different superscripts are significant at $p<0.05$ by Duncan's multiple range test.

immune system. After the mice immunized with type II collagen, the weight of spleen was increased due to promoting immune system via production of antibodies and immunoglobulin (Hongfang and Meng, 2011). The spleen index for the CIA group was significantly higher than those for the MTX and NPCC groups. Interestingly, the dietary supplement of nanopowdered peanut sprouts-added Caciocavallo cheese had the similar effects on the reduction of spleen index in the arthritis mice compared with the methotrexate. Based on the finding, it was suggested that dietary supplementation $(0.6 \mathrm{~g} / \mathrm{d})$ of nanopowdered peanut sprouts-added Caciocavallo cheese could inhibit the type II collagen-induced arthritis in mice.

Based on the results (clinical score, hind paw thickness, biochemical analysis, histological assessment, and spleen index) obtained from our study, there were no considerable differences between CC and NPCC models. In the present study, it was hypothesized that due to the advantage of size effect and high surface reactivity of nanoparticles, the addition of nanopowdered peanut sprouts would improve the reduction and treatment of arthritis symptom in the mice immunized with type II collagen. However, we did not find the more improved preventive effect of nanopowdered peanut sprouts-added Caciocavallo cheese on rheumatoid arthritis, as compared to Caciocavallo cheese. Accordingly, our study demonstrated that the addition of $0.5 \%(\mathrm{w} / \mathrm{v})$ of nanopowdered peanut sprouts into Cacio- cavallo cheese was not enough, so that the increase in the addition of nanopowdered peanut sprouts should be needed for the future study.

\section{Conclusion}

This study was designed to evaluate the influence of nanopowdered peanut sprouts-added Caciocavallo cheese on the prevention and treatment of rheumatoid arthritis in DBA/IJ mice immunized with type II collagen. Based on the findings (clinical score, paw thickness, serum analysis, and histological analysis), it is indicated that feeding nanopowdered peanut sprouts-added Caciocavallo cheese to the collagen-induced arthritis mice can reduce clinical score, paw thickness, TNF- $\alpha$, IL-1 $\beta$, IL- 6, IgG1, and histological scores in the mice. Therefore, the production of Caciocavallo cheese which incorporates nanopowdered peanut sprouts can broaden the utilization of peanut sprouts, and the products can be regarded as possible health-promoting nutraceutical foods, especially for the prevention and treatment of rheumatoid arthritis.

\section{Acknowledgements}

The present study was supported by a grant from Korea Institute of Planning and Evaluation for Technology in Food, Agriculture, Forestry and Fisheries in GyeonggiDo, Republic of Korea (111020-03-3-HD120).

\section{References}

1. Ahn, Y. J., Palanivel, G., and Kwak, H. S. (2012) Comparison of nanopowdered and powdered peanut sprout-added yogurt on its physicochemical and sensory properties during storage. Korean. J. Food Sci. An. 5, 553-560.

2. American Institute of Nutrition. (1977) Report of the American Institute of Nutrition Ad Hoc Committee on Standards for Nutritional Studies. J. Nutr. 107, 1340-1348.

3. Borchers, A. T., Keen, C. L., Cheema, G. S., and Gershwin, M. E. (2004) Theuse of methotrexate in rheumatoid arthritis. Semin. Arthritis Rheum. 34, 465-483.

4. Brand, D. D., Latham, K. A., and Rosloniec, E. F. (2007) Collagen-induced arthritis. Nat. Protoc. 2, 1269-1275.

5. Burns, J., Yokota, T., Ashihara, H., Lean, M. E., and Crozier, A. (2002) Plant foods and herbal sources of resveratrol. Food Chem. 50, 3337-3340.

6. Chunxia, C., Peng, Z., Huifang, P., Hanli, R., Zehua, H., and Jizhou, W. (2011) Extracts of Arisaemarhizomatum C.E.C. Fischer attenuate inflammatory response on collagen-induced arthritis in BALB/c mice. J. Ethnopharmacol. 133, 573-582.

7. Elmali, N., Baysal, O., Harma, A., Esenkaya, I., and Mizrak, 
B. (2006) Effects of resveratrol in inflammatory arthritis. Inflammation 30, 1-2.

8. Feldmann, M., Brennan, F. M., and Maini, R. N. (1996) Role of cytokines in rheumatoid arthritis. Annu. Rev. Immunol. 14, 397-440.

9. Gobbetti, M., Morea, M., Baruzzi, F., Corbo, M. R., Matarante, A., Considine, T., Cagno, R. D., Guinee, T., and Fox, P. F. (2002) Microbiological, compositional, biochemical and textural characterisation of Caciocavallo Pugliese cheese during ripening. Int. Dairy J. 12, 511-523.

10. Goldring, S. R. (2003) Pathogenesis of bone and cartilage destruction in rheumatoid arthritis. Rheumatology (Oxford).42, 11-16.

11. Hongfang, W. and Meng, Z. (2011) Immune function changes of rats in recremental graded exercise test. Afr. J. Microbiol. Res. 5, 1557-1561.

12. Lee, Y. K., Ahn, S. I., and Kwak, H. S. (2013) Optimizing microencapsulation of peanut sprout extract by response surface methodology. Food Hydrocolloid.30, 307-314.

13. Maini, R. N. and Taylor, P. C. (2000) Anti-cytokine therapy for rheumatoid arthritis. Annu. Rev. Med. 51, 207-229.

14. Mankin, H. J., Dorfman, H., Lippiello, L., and Zarins, A. (1971) Biochemical and metabolic abnormalities in articular cartilage from osteo-arthritic human hips. J. Bone Joint Surg. Am. 53, 523-537.

15. Park, H. S., Jeon, B. J., Ahn, J., and Kwak, H. S. (2007) Effects of nanocalcium supplemented milk on bone calcium metabolism in ovariectomized rats. Asian Austral. J. Anim. 20, 1266-1271.

16. Rasenack, N. and Muller, B. W. (2004) Micron-size drug particles: Common and novel micronization techniques. Pharm.
Dev. Technol. 9, 1-13.

17. Sánchez-Fidalgo, S., Cárdeno, A., Villegas, I., Talero, E., and de la Lastra, C. A. (2010) Dietary supplementation of resveratrol attenuates chronic colonic inflammation in mice. Eur. $J$. Pharmacol. 633, 78-84.

18. Stamp, L. K., James, M. J., and Cleland, L. G. (2005) Diet and rheumatoid arthritis: A Review of the literature. Semin. Arthritis Rheu. 35, 77-94.

19. Seo, M. H., Lee, S. Y., Chang, Y. H., and Kwak, H. S. (2009) Physicochemical, microbial and sensory properties of yogurt supplemented with nanopowdered chitosan during storage. J. Dairy Sci. 92, 5907-5916.

20. Smolen, J. S. and Steiner, G. (2004) Therapeutic strategies for rheumatoid arthritis. New Engl. J. Med. 350, 591-602.

21. Wang, K. H., Lai, Y. H., Chang, J. C., Ko, S. L., and Chiou, R. Y. (2005) Germination of peanut kernels to enhance resveratrol biosynthesis and prepare sprout as a functional vegetable. J. Agr Food Chem. 53, 242-246.

22. Xiong, Q. P., Zhang, Q. H., Zhang, D. Y., Shi, Y. Y., Jiang, C. X., and Shi, X. J. (2014) Preliminary separation and purification of resveratrol from extract of peanut (Arachishypogaea) sprouts by macroporous adsorption resins. Food Chem. 145, $1-7$.

23. Xuzhu, G., Komai-Koma, M., Leung, B. P., Howe, H. S., McSharry, C., Mclnnes, I. B., and Xu, D. (2012) Resveratrol modulates murine collagen-induced arthritis by inhibiting Th17 and B-cell function. Ann. Rheum. Dis. 71, 129-135.

24. Viji, V., Kavitha, S. K., and Helen, A. (2010) Bacopamonniera (L.) wettst inhibits type ii collagen-induced arthritis in rats. Phytother. Res. 9, 1377-1383.

(Received 2013.10.22/Revised 2014.1.8/Accepted 2014.1.10) 